\title{
Atlas of Ultrasound and Nerve Stimulation-Guided Regional Anesthesia
}

\author{
Ban C.H. Tsui. Consulting Editors: Vincent W.S. Chan, Brendan T. Finucane, \\ Thomas Grau, Anil H. Walji. Springer, 2007, 302 pp, \$179, ISBN 978-0-387-68158-0
}

\author{
Kishor Gandhi, MD • Thomas Kallarackal-Maliakal, MD • \\ Jeff Gadsden, MD · Admir Hadzic, MD, PhD
}

Published online: 19 December 2008

(C) Canadian Anesthesiologists' Society 2008

Regional anesthesia and peripheral nerve blocks have been revolutionized in the last few years by the introduction of monitoring of the relevant anatomy, needle placement, and disposition of local anesthetic using ultrasonography. Dr. Ban Tsui's text is one of the first to specifically focus on these and other significant developments in the field.

This 302-page colour atlas comprises 20 chapters, 289 quality pictures, 4 graphs, 78 anatomic drawings, 113 ultrasound images, and 30 tables. In the introductory chapters, attention is given to block room setup, regional anesthesia equipment, basics of electrophysiology, ultrasound imaging, and instrumentation. The author examines the structure and functionality of the equipment, and he gives recommendations on the choice of needles, stimulators, and many other important aspects of the clinical practice. Needle depth markings, stimulating catheters, echogenic needles, and the importance of injection pressure monitoring are also reviewed. Novices to ultrasound will find the chapter on Ultrasound Basics particularly useful, as it clearly outlines many aspects of ultrasound imaging, such as transducer choice, scanning frequency, optimizing needle visibility, and needle tracking.

The technique descriptions are prefaced by introductory chapters on clinical anatomy and are followed by descriptions of the most common peripheral nerve blocks and their variations. The chapters pertaining to techniques are logically and consistently organized to include clinical anatomy, patient positioning, sonographic imaging, needle insertion techniques, nerve stimulation, and local anesthetic application. An entire chapter is dedicated to the technique of catheter insertion using ultrasonography.

In addition to the nerve block techniques, the atlas includes additional areas relating to the author's expertise, such as epidural and caudal anesthesia using electrical nerve stimulation and ultrasound guidance. Separate chapters are dedicated to paravertebral and intercostal blocks. The atlas also features advanced ultrasound techniques, such as imaging and ultrasound-guidance of neuraxial blocks.

This text is nicely supplemented with numerous anatomical drawings and images of cadaveric dissections. Many chapters feature logically organized and easy to follow coloured tables. Ultrasound images are well labelled and are often accompanied by corresponding magnetic resonance images for easier understanding of the featured anatomy. The role of peripheral nerve stimulation in conjunction with ultrasound guidance is also examined, and practical management is recommended in various clinical scenarios. Relevant literature is cited throughout.

In conclusion, Atlas of Ultrasound and Nerve Stimulation-Guided Regional Anesthesia has a wealth of practical information for both students and seasoned anesthesiologists. As such, the atlas fills the vacuum in the literature on modern regional anesthesia techniques, and, as such, it should deserve a spot in every anesthesiologist's library.

K. Gandhi, MD - T. Kallarackal-Maliakal, MD .

J. Gadsden, MD · A. Hadzic, MD, PhD ( $\square)$

St. Luke's-Roosevelt Medical Center, New York, USA

e-mail: admir@nysora.com 